\title{
Multiplex-Real-Time-PCR
}

\section{Spezifisch - sicher - schnell: Nachweis von Influenzaviren}

\author{
DINAH HENRITZI, ANNIKA GRAAF, TIMM HARDER \\ FRIEDRICH-LOEFFLER-INSTITUT, GREIFSWALD - INSEL RIEMS
}

\section{Influenza viruses remain an imminent threat for animal and public health. Rapid and reliable diagnosis of acute infections are at the roots of mean- ingful control and prevention measures. Sensitive and highly specific reverse transcription real-time PCR (RT-qPCR) assays have revolutionized the diagnostic toolbox. RT-qPCRs for subtyping and pathotype characteri- zation of animal influenza viruses provide advantages in individual dia- gnosis and for long term monitoring programs.}

DOI: $10.1007 / \mathrm{s} 12268-019-1046-6$

(C) Springer-Verlag 2019

Als Goldstandard der Detektion von Viren als Krankheitserreger gilt klassischerweise der Nachweis infektiöser Erreger durch Virusisolierung in Versuchstieren oder in der Zellkultur. Oftmals sind diese Techniken kostenund zeitintensiv sowie im Ergebnis wenig sensitiv, da die Infektiosität im Probenmaterial rasch inaktiviert werden kann. Im Falle humanpathogener Erreger besteht beim Umgang mit einem infektionstüchtigen Virus auch ein Gefährdungspotenzial für das Laborpersonal; bei der Bearbeitung von vermehrungsfähigen Tierseuchenerregern muss die Umwelt vor diesen Pathogenen geschützt werden. Demgegenüber bietet der Nachweis von Bestandteilen eines Virus unabhängig von dessen Infektionstüchtigkeit etliche Vorteile. Die Polymerasekettenreaktion (PCR), ein hochsensitives und spezifisches Verfahren zum Nachweis von Genommaterial, ist eine der bedeutendsten methodischen Entwicklungen in der Molekularbiologie und hat seit den 1990er-Jahren Eingang in die Routine der infektiologischen Diagnostik gefunden. Die (semi-)quantitative Echtzeit-PCR (real-time quantitative PCR, RT-qPCR) stellt eine Weiterentwicklung des PCR-Prinzips dar (Abb. 1, [1]). RT-qPCRs erhöhen die Sicherheit der Erregeridentifizierung und ermöglichen die Quantifizierung von Erregergenomen in der Probe. Darüber hinaus ergeben sich eine Reihe technischer Vorteile, die diese Methode für kostengünstige und sichere klinische
Untersuchungen sowie für epidemiologisch orientierte Massenuntersuchungen interessant macht.

\section{Influenzaviren - Seuchenerreger und harmlose Begleiter}

Influenzaviren aus der Familie der Orthomyxoviridae sind behüllte, einzelsträngige RNAViren, deren Genom auf bis zu acht einzelne Gensegmente verteilt ist (segmentiertes Genom) [2]. Zurzeit kennt man vier genetisch definierte Genera: Influenza-A-Viren (IAV) mit einer Vielzahl verschiedener bei Vögeln und Säugern vorkommender Subtypen, Influenza-B-Viren (IBV) und -C-Viren (ICV), die den Menschen als wichtigste Wirtsspezies betreffen, ferner die erst vor Kurzem bei Rindern und sporadisch bei Schweinen entdeckten Influenza-D-Viren (IDV) [3]. ICV und IDV scheinen kaum klinische Bedeutung zu besitzen. Dagegen sind IAV und IBV wichtige Pathogene, die bei Menschen Erreger der „echten“ Grippe sind. Grippe bleibt auch weiterhin eine der volkswirtschaftlich bedeutsamsten Virusinfektionen des Menschen, die alljährlich allein in Deutschland Millionen von Infektionen, Hunderttausende Krankschreibungen und saisonabhängig Tausende tödlich endender Erkrankungen bedingt [4]. IAV-Infektionen entfalten auch bei Wild- und Haustieren große Bedeutung. Sie sind in der Geflügelhaltung als Ursache verheerender Seuchenzüge gefürchtet und führen auch in der Schweinehaltung zu wirtschaftlich bedeutenden Verlusten [5, 6]. Sporadisch können einige Virusstämme auch von Vögeln oder Schweinen auf Menschen übertragen werden (zoonotische Influenzaviren). Unter für das Virus günstigen Umständen kann aus solchen Zufallsübertragungen eine weltweite Epidemie, eine Pandemie, in der menschlichen Population resultieren [4].

IAV werden aufgrund der Merkmale ihrer Oberflächenglykoproteine, Hämagglutinin (HA) und Neuraminidase (NA), in Subtypen untergliedert [2]. Derzeit werden $18 \mathrm{HA}$ - und elf NA-Subtypen unterschieden. In einem einzelnen Viruspartikel ist dabei jeweils ein HASubtyp mit einem NA-Subtyp kombiniert, sodass der Subtyp nach dem Schema $\mathrm{H}[\mathrm{x}=1-18], \mathrm{N}[\mathrm{y}=1-11]$ benannt werden kann. Aufgrund des segmentierten Genoms kann es zu einem Austausch von Gensegmenten zwischen zwei IAV kommen, wenn diese die gleiche Zelle infizieren. Dieser Austauschvorgang wird als Reassortierung bezeichnet und kann jedes der Gensegmente einzeln oder in Gruppen betreffen [2]. Sind von der Reassortierung die HA- und/oder NASegmente betroffen, können neue HA/NAKombinationen (= Subtypen) entstehen. Die größte Vielfalt von Subtypen, H1-16 und N1-9, findet sich in Wildvogelpopulationen (aviäre Influenzaviren, AIV), die sich dort hocheffektiv vor allem im Darm-, aber auch im Atemtrakt der Wirte vermehren, jedoch meist keine oder lediglich milde Krankheitserscheinungen hervorrufen (niedrigpathogene AIV). Sporadisch können AIV aus diesem Reservoir auf andere Tiere übertragen werden. Erfolgt in der neuen Wirtsspezies eine zufallsgesteuerte Adaptation der Viren, können sich stabile Infektionsketten etablieren, und das Virus beginnt unabhängig vom ehemaligen Reservoir zu zirkulieren (Abb. 2). Die Subtypen H17N10 und H18N11 wurden bislang nur in Fledermäusen nachgewiesen.

Ein weiterer Faktor der Vielfalt von Influenza-A- und -B-Viren liegt in ihrer hohen Punktmutationsfrequenz [2]. Ein stetes Wechsel- 


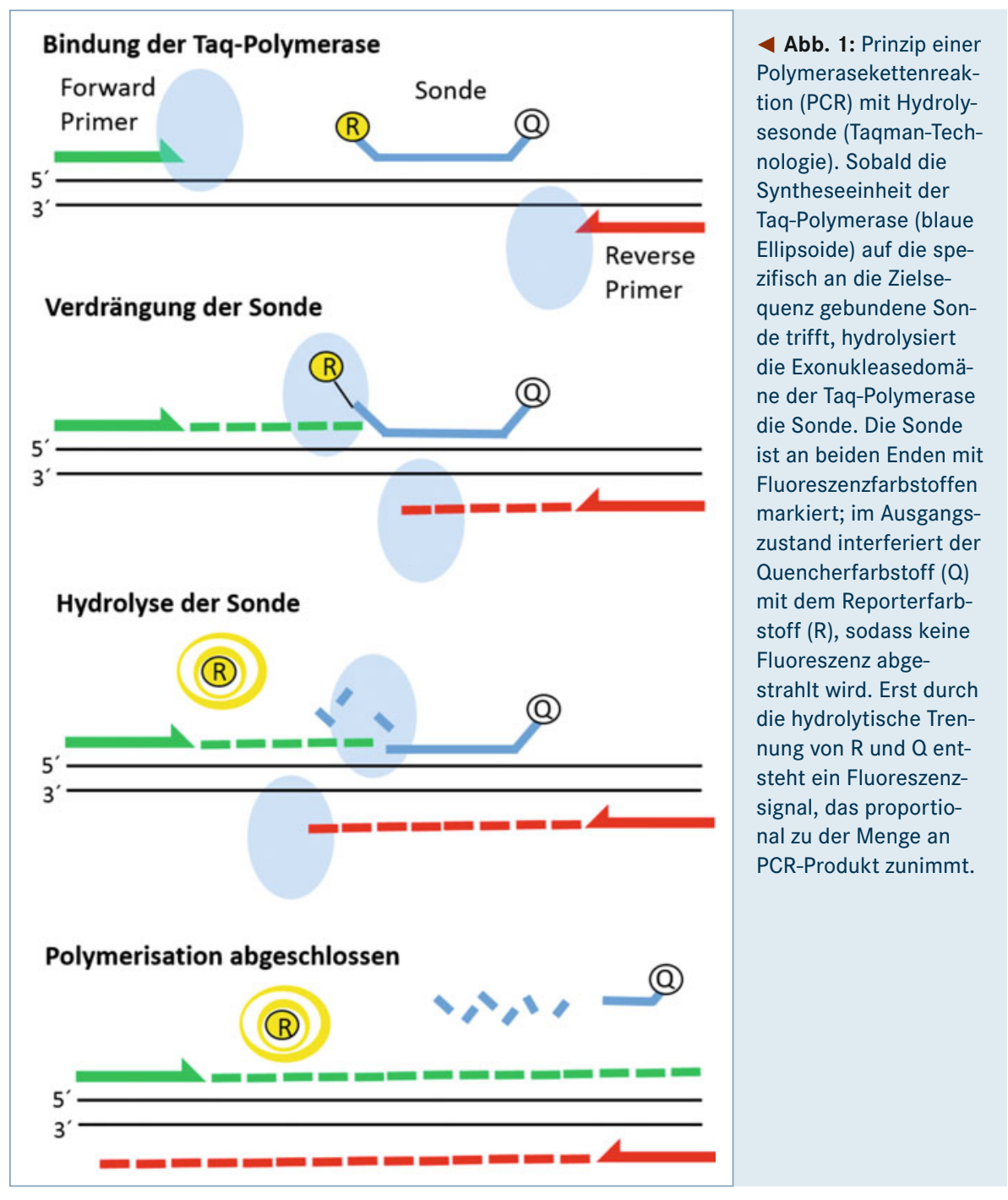

spiel zwischen der Entstehung immer neuer, geringgradig veränderter Varianten und dem äußeren Selektionsdruck, z. B. durch die Populationsimmunität der Wirtsspezies, steuert die jährliche saisonale Grippeepidemie des Menschen [7]. Bei den AI-Viren der Subtypen $\mathrm{H} 5$ und $\mathrm{H} 7$ kann die Mutationsfreudigkeit zur Entstehung hochgradig krankmachender Varianten führen (Abb. 2, rot hervorgehoben). Hierbei entstehen spontan aus niedrigpathogenen (low pathogenic, LP) Vorläufern hochpathogene (highly pathogenic, HP) Erreger, welche die mit sehr hoher Mortalität bei Geflügel verlaufende klassische Geflügelpest auslösen und daher tierseuchenrechtlich weltweit gemaßregelt werden [2]. HPAIV können mit infiziertem Geflügel verbreitet werden. Bei Übertragungen auf Populationen migrierender Wildvögel kann das Virus mit den Zugbewegungen dieser Vögel in kurzer Zeit über längere Distanzen verbreitet werden. Bestimmte HPAIV können auch auf Menschen übertragen werden und schwere, nicht selten tödlich verlaufende Infektionen auslösen [8].

\section{Variabilität der Influenzaviren - Herausforderungen für Immunsysteme und Diagnostiker}

Das breite Spektrum der Genera, Sub- und Pathotypen sowie Einzelvarianten des Influenzavirus stellt die Diagnostik vor enorme Herausforderungen. Zum Nachweis akuter Influenzainfektionen bei Tieren wurden diagnostische Werkzeuge auf Basis der beschriebenen RT-qPCR-Technologie entwickelt, die eine Ablösung der klassischen Virusdiagnostik eingeleitet haben [9]. Auslöser entsprechender Arbeiten am FriedrichLoeffler-Institut (FLI) war 2006 der erstmalige Einbruch von zoonotischen HPAI-Viren des Subtyps H5N1 aus Ostasien nach Europa. In den Folgejahren kam es wiederholt zu Eintragungen verwandter HPAI-Viren, die 2016/17 in einer massiven Epidemie bei Wildvögeln und Geflügel in Europa gipfelten [10].
Effektive Kontrollmaßnahmen setzen genaue Kenntnisse über den Erreger und dessen Verbreitung voraus. Daher ist eine akkurate Diagnostik stets Voraussetzung einer erfolgreichen Tierseuchenkontrolle.

Die Diagnostik von HPAIV-Infektionen umfasst drei Schritte: Nachweis eines IAV (Screening), Subtypdifferenzierung und Pathotypanalyse (sofern im vorigen Schritt die Subtypen H5/H7 nachgewiesen wurden). Abbildung 3 vergleicht klassische Verfahren und neu entwickelte RT-qPCR-Techniken. RT-qPCR-basierte Methoden verkürzen die Zeit bis zum finalen Befund von zwei bis drei Wochen auf einen Arbeitstag. Die Reaktionszeit des Tierseuchenkontrollapparates zwischen der Äußerung eines Infektionsverdachts und dessen diagnostischer Abklärung soll so kurz wie möglich sein, damit geeignete Kontroll- und Präventionsmaßnahmen unverzüglich eingeleitet werden können. Darüber hinaus sparen RT-qPCRs Tierversuche ein (Pathogenitätstest, Serumherstellung), sind tauglich für Massenuntersuchungen und reduzieren die Gefährdung des bearbeitenden Personals beträchtlich. Die in der RT-qPCR zur Pathotypisierung eingesetzten Oligonukleotidsonden (Abb. 3A) zielen dabei auf einen Bereich des HA-Gens ab, der für eine endoproteolytische Schnittstelle, eine Sollbruchstelle, des HA-Proteins codiert. Die Ausgestaltung dieses Bereiches bestimmt die Pathogenität (LP versus HP) von H5- und H7-Viren wesentlich [6]. Die Variabilität dieses Bereiches erforderte erheblichen Forschungsaufwand, um geeignete Sonden zu definieren, die LP- und HP-typische Nukleotidsequenzen in diesem Bereich sicher differenzieren (Abb. 3A, [11, 12]). Obwohl noch Einschränkungen in der diagnostischen Breite zu berücksichtigen sind, stellen die pathotypspezifischen RT-qPCRs eine wichtige Alternative zu tierversuchs- und sequenzierungsabhängigen Pathotypisierungen dar.

IAV-Infektionen bei Säugetieren beschränken sich auf wenige Subtypen. Im Menschen sowie beim Schwein sind es derzeit weltweit H1, H3 und N1, N2 [4, 7]. Übertragungen zwischen Mensch und Schwein kommen in beiden Richtungen vor; die jüngste humane Pandemie wurde 2009 durch ein im Schwein zirkulierendes (porzines) H1N1-Virus ausgelöst [8]. In Schweinebeständen führen IAV-Infektionen weltweit zu hoher Morbidität, allerdings zu geringer Mortalität [6]. Akute Atemwegsbeschwerden stehen hierbei im Vordergrund und bedingen teilweise beträchtliche wirtschaftliche Schäden. In Europa zirkulie- 


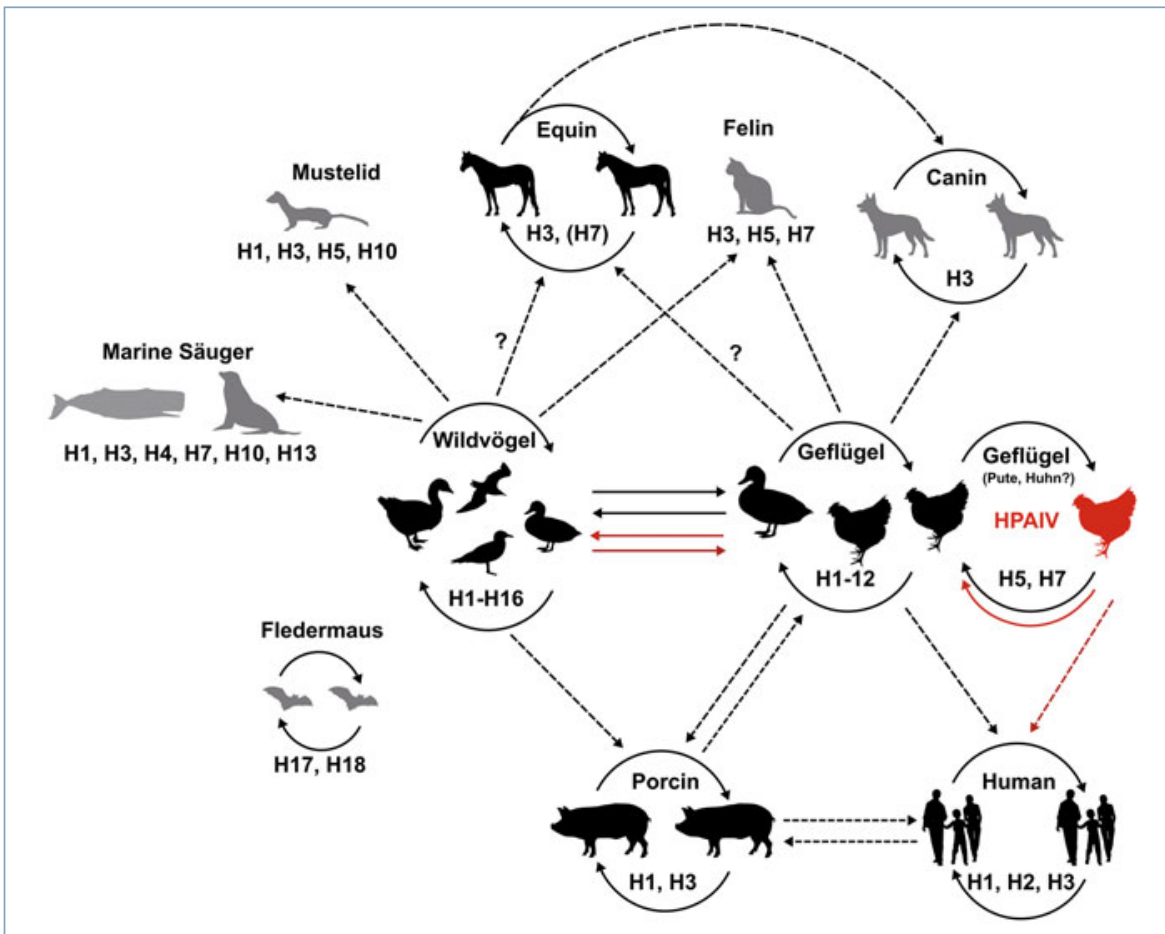

$\Delta$ Abb. 2: Wildvögel bilden ein Reservoir der Variationsbreite von Influenza-A-Viren. Sporadisch (gestrichelt) können Viren von dort auf andere Tiere übertragen werden, sich adaptieren und unabhängig vom ehemaligen Reservoir zirkulieren (durchgezogen). Viren der Subtypen H5 und H7 können in Hühnervögeln spontan zu einer hochpathogenen Form (HPAIV) mutieren (rot), die auch ein Risiko für den Menschen darstellen kann [10]. ren beim Schwein die Subtypen H1N1, H1N2 und H3N2 [13]. Aufgrund ihrer unterschiedlichen phylogenetischen und antigenetischen Eigenschaften unterteilt man die H1-Subtypen nach ihrem Ursprung in aviäre (av), pandemische (pdm) und human-saisonale (hu) sowie die N1-Subtypen in aviäre (av) und pandemische (pdm) [14]. Daraus ergeben sich vier Hauptsubtypen: H1avNav, H1pdmN1pdm, H1huN2 und H3N2; Reassortierungen zwischen diesen vier Subtypen verkomplizieren die Lage weiter. Die Grundlage der Diagnostik sind auch hier von uns neu entwickelte Multiplex-RT-qPCR-abhängige Werkzeuge [14, 15]. Hierzu wurden RT-qPCRs verschiedener Spezifität in einer Reaktion vereinigt (Abb. 3B). Die gegenüber herkömmlichen Verfahren der Virusisolierung erhöhte Sensitivität der spezifischen RT-qPCRs für Influenzaviren der Typen A, B, C und D sowie für porzine IAV-Subtypen ermöglicht die direkte Analyse und Subtypisierung von klinischen Proben (Abb. 3B). Aufgrund der Massentauglichkeit der Multiplexverfahren sind auch ausgedehnte epidemiologische Studien möglich, die neue Einsichten in die Entstehung und Verbreitung porziner IAV-Infektionen mit zoonotischem Potenzial liefern.
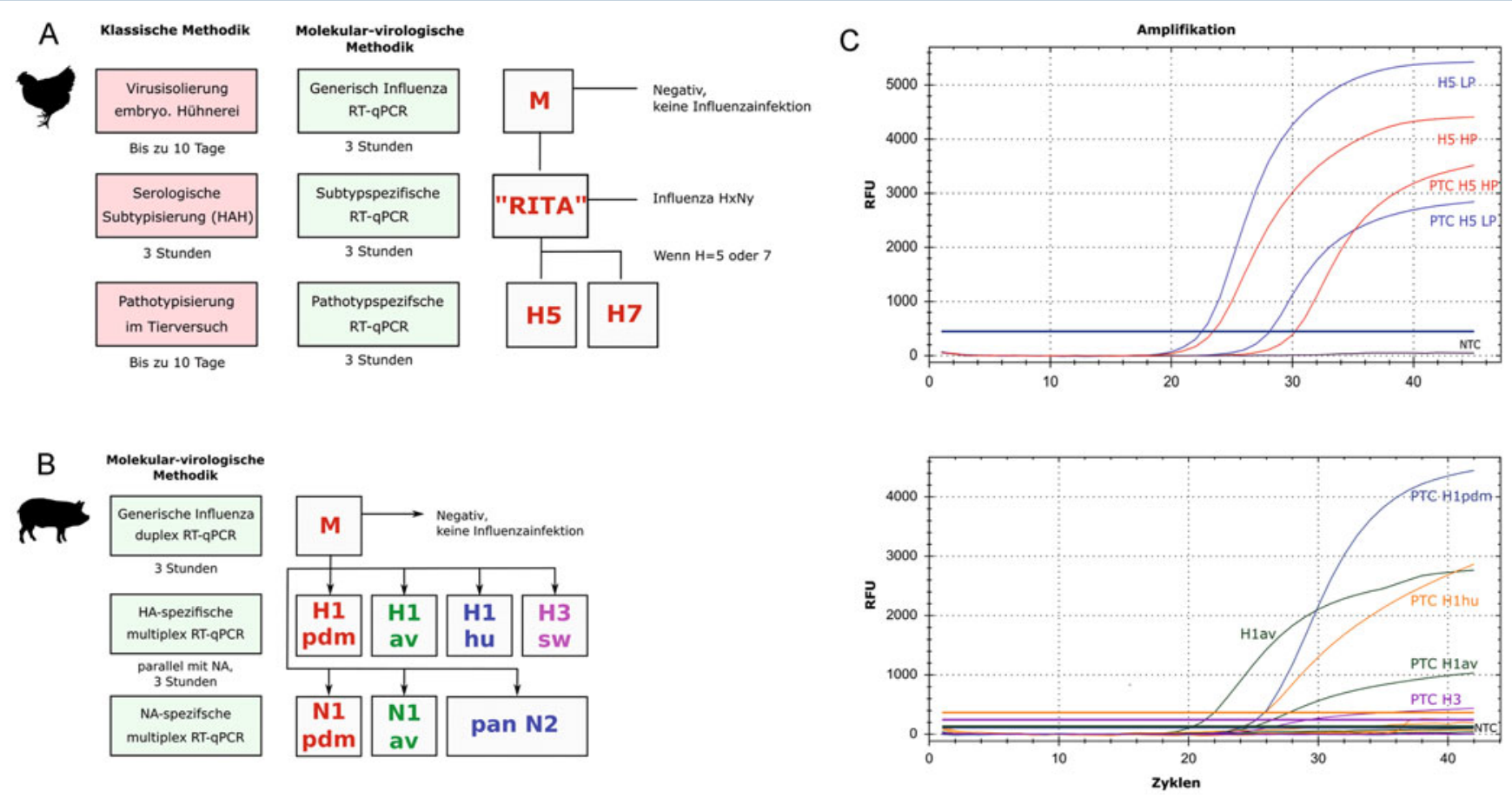

$\Delta$ Abb. 3: A, Die dreistufige RT-qPCR-basierte Diagnostik von aviären Influenzavirusinfektionen bietet hinsichtlich einer Zeitersparnis und erhöhter Sensitivität entscheidende Vorteile gegenüber klassischen Verfahren [9, 11, 12, 16], M: matrixgenspezifische generische RT-qPCR; RITA: Riems influenza A typing array für 16 HA- und neun NA-Subtypen. B, Auch der diagnostische Algorithmus zum Nachweis und zur Subtypisierung von Influenzavirusinfektionen bei Schweinen profitiert durch die Einführung von Multiplex-RT-qPCRs [14, 15]. C, In den Kurvendiagrammen wird die Zunahme der Fluoreszenz in positiven Proben gemessen. Oben: Nachweis des LP- und HP-Phänotyps von AIV des Subtyps H5; unten: Differenzierung von vier HA-Subtypen von porzinen Influenzaviren. 
Die am FLI erarbeiteten RT-qPCR-Methoden stellen wichtige Optimierungen der Diagnostik von Influenzainfektionen bei Wildund Haustieren dar und erleichtern die Durchführung von epidemiologisch aussagekräftigen, längerfristigen Überwachungsprogrammen [11, 12, 14-16]. Laufende Anpassungen an etwaig neu auftretende Varianten und Pathotypen bleiben essenziell, um eine hohe Sensitivität und Spezifität über die Zeit hin zu gewährleisten. RT-qPCR-Techniken sind auch in der Diagnostik anderer Tierseuchen zum Standard geworden.

\section{Danksagung}

D. Henritzi wurde im Rahmen des europaweiten passiven Surveillanceprogramms „Porzine Influenza“ am Friedrich-Loeffler-Institut (FLI) (Ri-0402, IDT Biologika GmbH) gefördert. A. Graaf wurde aus intramuralen Forschungsmitteln des FLI gefördert (HR-0008).

\section{Literatur}

[1] Arya M, Shergill IS, Williamson M et al. (2005) Basic principles of real-time quantitative PCR. Exp Rev Mol Diagn 5:209-219

[2] Cheung TK, Poon LL (2007) Biology of influenza A virus. In: Biology of Emerging Viruses: Sars, Avian and Human Influenza, Metapneumovirus, Nipah, West Nile, and Ross River Virus. Lal SK (Hrsg). Annals of the New York Academy of Sciences 1102:1-25

[3] Su S, Fu XL, Li GR et al. (2017) Novel influenza D virus: epidemiology, pathology, evolution and biological characteristics. Virulence 8:1580-1591

[4] Paules C, Subbarao K (2017) Influenza. Lancet 390:697708

[5] Franca MS, Brown JD (2014) Influenza pathobiology and pathogenesis in avian species. Curr Top Microbiol Immunol 385: 221-242

[6] Rajao DS, Anderson TK, Gauger PC et al. (2014)

Pathogenesis and vaccination of influenza A virus in swine. Curr Top Microbiol Immunol 385:307-326

[7] Petrova VN, Russell CA (2018) The evolution of seasonal influenza viruses. Nat Rev Microbiol 16:47

[8] Short KR, Richard M, Verhagen JH et al. ( 2015) One health, multiple challenges: the inter-species transmission of influenza A virus. One Health 1:1-13

[9] Spackman E, Suarez DL (2008) Avian influenza virus. Type A influenza virus detection and quantitation by realtime RT-PCR. Methods Mol Biol 436:19-26
[10] Globig A, Staubach C, Sauter-Louis C et al. (2018) Highly pathogenic avian influenza H5N8 clade 2.3.4.4b in Germany in 2016/2017. Front Vet Sci 4:240

[11] Graaf A, Beer M, Harder T (2017) Real-time reverse transcription PCR-based sequencing-independent pathotyping of Eurasian avian influenza A viruses of subtype H7. Virol J $14: 137$

[12] Naguib MM, Graaf A, Fortin A et al. (2017) Novel realtime PCR-based patho- and phylotyping of potentially zoonotic avian influenza A subtype $\mathrm{H} 5$ viruses at risk of incursion into Europe in 2017. Eurosurveillance 22:15-26

[13] Anderson TK, Macken CA, Lewis NS et al. (2016) A phylogeny-based global nomenclature system and automated annotation tool for $\mathrm{H} 1$ hemagglutinin genes from swine influenza A viruse. mSphere 1:e00275-16

[14] Henritzi D, Hoffmann B, Wacheck S et al. (2019) A newly developed tetraplex real-time RT-PCR for simultaneous screening of influenza virus types A, B, C and D. Influenza Other Respir Viruses 13:71-82

[15] Henritzi D, Zhao N, Starick E et al. (2016) Rapid detection and subtyping of European swine influenza viruses in porcine clinical samples by haemagglutinin- and neuraminidase-specific tetra- and triplex real-time RT-PCRs. Influenza Other Respir Viruses 10:504-517

[16] Hoffmann B, Hoffmann D, Henritzi D et al. (2016) Riems influenza a typing array (RITA): an RT-qPCR-based low density array for subtyping avian and mammalian influenza a viruses. Sci Rep 6:27211

\section{Korrespondenzadresse:}

Prof. Dr. Timm Harder

Friedrich-Loeffler-Institut

Institut für Virusdiagnostik

Südufer 10

D-17493 Greifswald

Tel.: 038351-7-1152/1546

Timm.Harder@fli.de

\begin{tabular}{|c|c|}
\hline \multicolumn{2}{|l|}{ AUTOREN } \\
\hline & $\begin{array}{l}\text { Dinah Henritzi } \\
\text { 2005-2013 Biologiestudium (Diplom) an der Rheinischen Friedrich-Wilhelms-Univer- } \\
\text { sität Bonn. 2015-2018 Promotion und anschließend Postdoc am Friedrich-Loeffler-In- } \\
\text { stitut, Insel Riems, unter der Leitung von Prof. Dr. T. Harder. }\end{array}$ \\
\hline & $\begin{array}{l}\text { Annika Graaf } \\
\text { 2010-2016 Veterinärmedizinstudium an der Ludwig-Maximilians-Universität Mün- } \\
\text { chen. 2016-2019 Promotion und Postdoc am Friedrich-Loeffler-Institut, Insel Riems, } \\
\text { unter der Leitung von Prof. Dr. T. Harder. }\end{array}$ \\
\hline $\mathrm{A}$ & $\begin{array}{l}\text { Timm Harder } \\
\text { 1983-1989 Tiermedizinstudium an der Tierärztlichen Hochschule Hannover; dort } \\
\text { 1989-1993 Promotion und Postdoc. 1994-1996 Postdoc an der Erasmus Universi- } \\
\text { teit, Rotterdam; Postdoc an der Christian-Albrechts-Universität Kiel (Habilitation und } \\
\text { apl. Professur). Seit } 2006 \text { am Friedrich-Loeffler-Institut, Insel Riems, Leiter des Na- } \\
\text { tionalen Referenzlabors für Aviäre Influenza und Experte für Influenzainfektionen der } \\
\text { Tiere bei der Welttiergesundheitsorganisation (O. I. E.). }\end{array}$ \\
\hline
\end{tabular}

